\title{
SKY BRIGHTNESS AND TRANSPARENCY IN THE $i$-BAND AT DOME A, ANTARCTICA
}

\author{
Hu Zou ${ }^{1,2}$, Xu Zhou ${ }^{1,3}$, Zhaoji Jiang ${ }^{1,3}$, M. C. B. Ashley ${ }^{4}$, Xiangqun Cui $^{3,5}$, Longlong Feng ${ }^{3}$, Xuefei Gong ${ }^{3,5}$, \\ Jingyao Hu ${ }^{1,3}$, C. A. Kulesa ${ }^{6}$, J. S. Lawrence ${ }^{7,8}$, Genrong Liu ${ }^{5}$, D. M. Luong-Van ${ }^{4}$, Jun Ma ${ }^{1}$, A. M. Moore $^{9}$, \\ C. R. Pennypacker ${ }^{10}$, Weijia Qin ${ }^{11}$, Zhaohui Shang ${ }^{12}$, J. W. V. Storey ${ }^{4}$, Bo Sun ${ }^{11}$, T. Travouillon ${ }^{10}$, C. K. Walker $^{6}$,

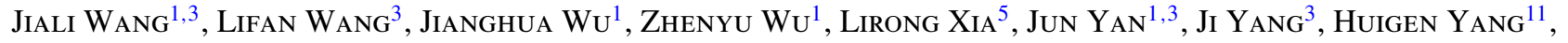 \\ YongQiang YaO ${ }^{1}$, Xiangyan Yuan ${ }^{3,5}$, D. G. York ${ }^{13}$, Zhanhai Zhang ${ }^{11}$, And ZhenXi Zhu ${ }^{3}$ \\ ${ }^{1}$ National Astronomical Observatories, Chinese Academy of Sciences, Beijing 100012, China; zhouxu@bao.ac.cn \\ ${ }^{2}$ Graduate University of Chinese Academy of Sciences, Beijing 100049, China \\ ${ }^{3}$ Purple Mountain Observatory, Chinese Academy of Sciences, Nanjing 210008, China \\ ${ }^{4}$ School of Physics, University of New South Wales, NSW 2052, Australia \\ ${ }^{5}$ Nanjing Institute of Astronomical Optics and Technology, Nanjing 210042, China \\ ${ }^{6}$ Steward Observatory, University of Arizona, Tucson, AZ 85721, USA \\ ${ }^{7}$ Department of Physics, Macquarie University, NSW 2109, Australia \\ ${ }^{8}$ Anglo-Australian Observatory, NSW 1710, Australia \\ ${ }^{9}$ Department of Astronomy, California Institute of Technology, Pasadena, CA 91125, USA \\ ${ }^{10}$ Lawrence Berkeley National Laboratory, Berkeley, CA 94720, USA \\ ${ }^{11}$ Polar Research Institute of China, Pudong, Shanghai 200136, China \\ 12 Tianjin Normal University, Tianjin 300074, China \\ ${ }^{13}$ Department of Astronomy and Astrophysics and Enrico Fermi Institute, University of Chicago, Chicago, IL 60637, USA \\ Received 2009 December 2; accepted 2010 June 19; published 2010 July 12
}

\begin{abstract}
The $i$-band observing conditions at Dome A on the Antarctic plateau have been investigated using data acquired during 2008 with the Chinese Small Telescope Array. The sky brightness, variations in atmospheric transparency, cloud cover, and the presence of aurorae are obtained from these images. The median sky brightness of moonless clear nights is $20.5 \mathrm{mag} \operatorname{arcsec}^{-2}$ in the SDSS $i$ band at the south celestial pole (which includes a contribution of about 0.06 mag from diffuse Galactic light). The median over all Moon phases in the Antarctic winter is about $19.8 \mathrm{mag} \mathrm{arcsec}^{-2}$. There were no thick clouds in 2008. We model contributions of the Sun and the Moon to the sky background to obtain the relationship between the sky brightness and transparency. Aurorae are identified by comparing the observed sky brightness to the sky brightness expected from this model. About $2 \%$ of the images are affected by relatively strong aurorae.
\end{abstract}

Key words: methods: data analysis - methods: statistical - techniques: photometric - telescopes

\section{INTRODUCTION}

In selecting observatory sites for ground-based optical/ IR astronomy, some of the most important considerations are the night-sky brightness, transparency, seeing, number of clear nights, humidity, and photometric stability. The Antarctic plateau offers some attractive advantages for ground-based astronomical observations. Site testing over the past decade has revealed that Antarctica, relative to temperate latitude observatories, has lower infrared sky brightness, better freeatmosphere seeing, greater transparency, a lower turbulent boundary layer, and much lower water vapor content (see, e.g., reviews by Aristidi et al. 2005a, Burton et al. 2005, and Storey et al. 2007). About half the area of the Antarctic continent is at an elevation of over $3000 \mathrm{~m}$ above the sea level, and the year-round average temperature on the plateau is about $-50^{\circ} \mathrm{C}$, dropping to below $-80^{\circ} \mathrm{C}$ on occasion. The average of the water vapor column over the plateau has been predicted to be less than $250 \mu \mathrm{m}$ (Chamberlin 2001) and has been measured at Dome A to be below $100 \mu \mathrm{m}$ for $25 \%$ of the time (Yang et al. 2010). Wind speeds at the summits of the plateau are very low close to the surface and the median thickness of the turbulent boundary layer has been measured at Dome A as $13.9 \mathrm{~m}$ (Bonner et al. 2010). These exceptionally favorable geographical and meteorological conditions result in substantial advantages for millimeter, submillimeter, infrared, and optical astronomy.

Several large astronomical facilities are already operating at some sites on the Antarctic plateau; e.g., the South Pole
Telescope (Ruhl et al. 2004) at the US Amundsen-Scott station. Proposed facilities include a $2.5 \mathrm{~m}$ optical telescope, PILOT (now called PLT) at Concordia Station, Dome C (Storey et al. 2008; Lawrence et al. 2009a), and a $2 \mathrm{~m}$ class infrared telescope planned for deployment to Dome Fuji by Japan (Takato et al. 2008).

At Dome C (3250 m elevation), the average seeing is 0.27 above a $\sim 30 \mathrm{~m}$ ground layer and the average surface wind speed is $2.9 \mathrm{~m} \mathrm{~s}^{-1}$ (Lawrence et al. 2004; Aristidi et al. 2005a, 2005b). Dome A is the highest point of the continent, with an elevation of $4093 \mathrm{~m}$. It might be reasonably predicted that Dome A could be as good as or even a better astronomical site than Dome $\mathrm{C}$, with better seeing, higher transparency, and thinner surface layer. Saunders et al. (2009) compared the sites Dome A, B, C, F and Ridge A and B in their cloud cover, free-atmosphere seeing, precipitable water vapor, temperature, and auroral emission, and concluded that, overall, Dome A might be the best of the existing bases for astronomical observations.

Following the success of the first Chinese expedition team to Dome A in 2005 January, the Chinese Center for Antarctic Astronomy (CCAA) began planning for the deployment of a telescope array called Chinese Small Telescope ARray (CSTAR; Yuan et al. 2008), which is described in the following section.

In this paper, we use CSTAR images obtained during 2008 to assess the night sky brightness at Dome A. Both the variation in atmospheric transparency and cloud cover are also derived. We then analyze the contribution to the sky brightness from the Sun and the Moon and investigate the correlation 
between sky brightness and atmospheric transparency. Finally, the contribution from aurorae is assessed.

\section{INSTRUMENT AND OBSERVATIONS}

The CSTAR telescope array was built by the Nanjing Institute of Astronomical Optics \& Technology (NIAOT) and consists of four co-aligned Schmidt telescopes on a fixed mounting pointing toward the south celestial pole. Each telescope has a different filter: $g, r, i$, and open. The detectors are Andor DV435 $1 \mathrm{k} \times 1 \mathrm{k}$ CCDs with $13 \mu \mathrm{m}$ pixels, giving a plate scale of $15^{\prime \prime} \mathrm{pixel}^{-1}$. The entrance pupil diameter is $145 \mathrm{~mm}$ (effective aperture: $100 \mathrm{~mm}$ ) and the field of view is about $20 \mathrm{deg}^{2}$. The scientific goals for CSTAR were primarily the detection of variable stars and measurements of sky brightness, the stability of the atmospheric transparency, and cloud cover.

The CSTAR telescopes, cameras, and computer system were tested under conditions of very low temperature, low air pressure, and long-duration continuous operation. The observational testing of the array was done at the Xinglong Station of National Astronomical Observatories of China (NAOC; Zhou et al. 2010a).

In 2008 January, CSTAR was transported to Dome A and successfully installed, with the intention of making observations of the south celestial pole region from 2008 April to August. Power, heat, and internet connectivity for CSTAR were provided by PLATO (the PLATeau Observatory), developed by the University of New South Wales (Yang et al. 2009; Lawrence et al. 2009a)

After deployment to Dome A, only the $i$-band telescope (CSTAR\#1) operated well, observing throughout the winter with just a few short interruptions; the other three telescopes had either small problems with their optical alignment or their control computers. However, all four telescopes operated sufficiently well to allow us to derive some information on sky conditions in the other filter bands, and this analysis will be published in a subsequent paper. Over 310,000 images were obtained, with integration times of $20 \mathrm{~s}$ or $30 \mathrm{~s}$, between 2008 March 4 and 2008 August 8, giving a total exposure time on the sky of about $1728 \mathrm{hr}$ (Zhou et al. 2010b). While CSTAR was observing, roughly one-third of the catalogs and one original image were transferred via Iridium communication satellite each day. All the catalogs and images were brought back by the 25th Chinese Research Expedition Team in 2009 April. The catalogs contain the ambient temperature, observing date and time, exposure time and background brightness, plus the instrumental magnitudes in three different apertures for all detected point sources calculated by an automatic aperture photometry pipeline. For each image about 10,000 sources down to $i \sim 16$ mag were detected. Several variable stars were identified and the complete observational catalog has now been released (Zhou et al. 2010b). As the catalog has continuous observations over several months, the data are ideal for the detection and monitoring of variable stars (J. H. Wu et al. 2010, in preparation).

Observations began on 2008 March 4 and ended on 2008 August 8, a few weeks earlier than expected, due to a power failure. Images were obtained whenever the Sun elevation was sufficiently below the horizon. There were two time periods during which no observations were made due to issues with the computer system: 2008 May 19-29 and 2008 June 30 to 2008 July 15 .

\section{RESULTS AND DISCUSSIONS}

\subsection{Sources of Sky Brightness}

Night sky brightness is one of the key parameters for qualifying a site for ground-based astronomical observation. Many sources contribute to the sky background including scattering from the Sun and the Moon, airglow, zodiacal light, aurorae, star light and interstellar dust scattering, extragalactic light, and artificial light contamination (Leinert et al. 1997; Benn \& Ellison 1998a). The contributions of these sources to the sky brightness at Dome $\mathrm{C}$ have been reviewed by Kenyon \& Storey (2006) and this is generally applicable to Dome A.

Artificial light pollution is essentially non-existent in Antarctica. The main contribution to the sky background is usually from the atmospheric scattering of the light of the Sun and the Moon. Benn \& Ellison (1998a) showed that zodiacal light (i.e., sunlight scattered by interplanetary dust) may contribute up to half the intrinsic sky brightness when the Sun and the Moon are down. However, this is not an issue for CSTAR observations since the field that we are observing is centered on the south celestial pole, with ecliptic longitude $\lambda=270^{\circ}$ and latitude $\beta=-66.6$, and is far from the region affected by zodiacal light. On the other hand, we expect an increased contribution from the Milky Way, since the Galactic latitude $b$ of the south celestial pole is $-27^{\circ}$, relatively close to the Galactic plane. We quantify this effect in Section 3.3.

Another important source of light is from the aurorae, which are common in polar regions. Aurorae are generated from electrons from the solar wind that are trapped in the upper atmosphere by the magnetic field of the Earth. The intensity and frequency of occurrence of aurorae depend upon the solar activity. From satellite images and ground-based observations, aurorae are known to be concentrated into an oval-shaped annulus centered on the geomagnetic south pole. As noted by Dempsey et al. (2005), the more intense the solar activity, the larger the extent of the oval. Domes $\mathrm{A}$ and $\mathrm{C}$ both lie within the "hole" in the center of this oval-shaped region and so are less seriously affected by aurorae than sites lying on the oval itself. In 2008, solar activity was close to its minimum level so that, perhaps paradoxically, Dome A would have experienced more aurorae than in an average year. The maximum of solar cycle 24 is predicted to appear in 2012 December (Kilcik et al. 2009), so the number and intensity of aurorae appearing above Dome A should then be at a minimum.

To better understand the effect of the Sun, the Moon, and cloud on the sky brightness, we modeled the contributions of the Sun and the Moon light without the influence of clouds in Sections 3.6 and 3.8. After removing these two sources of sky light, we consider the relationship between the residual sky background and atmospheric transparency (see Section 3.9). The remaining variable component of the sky brightness can then be attributed to aurorae (see Section 3.10).

\subsection{CSTAR Measurements of Sky Brightness}

The catalogs processed by the CSTAR pipeline contain the sky background values in analog-to-digital converter counts (ADU). The instrumental magnitude, $m$, in the CSTAR photometric system is defined as

$$
m_{\mathrm{CSTAR}}=-2.5 \log (\mathrm{ADU})+25 \text {. }
$$

Zhou et al. (2010b) used the USNO-B1 (U.S. Naval Observatory) star catalog to flux calibrate the CSTAR images and derived 


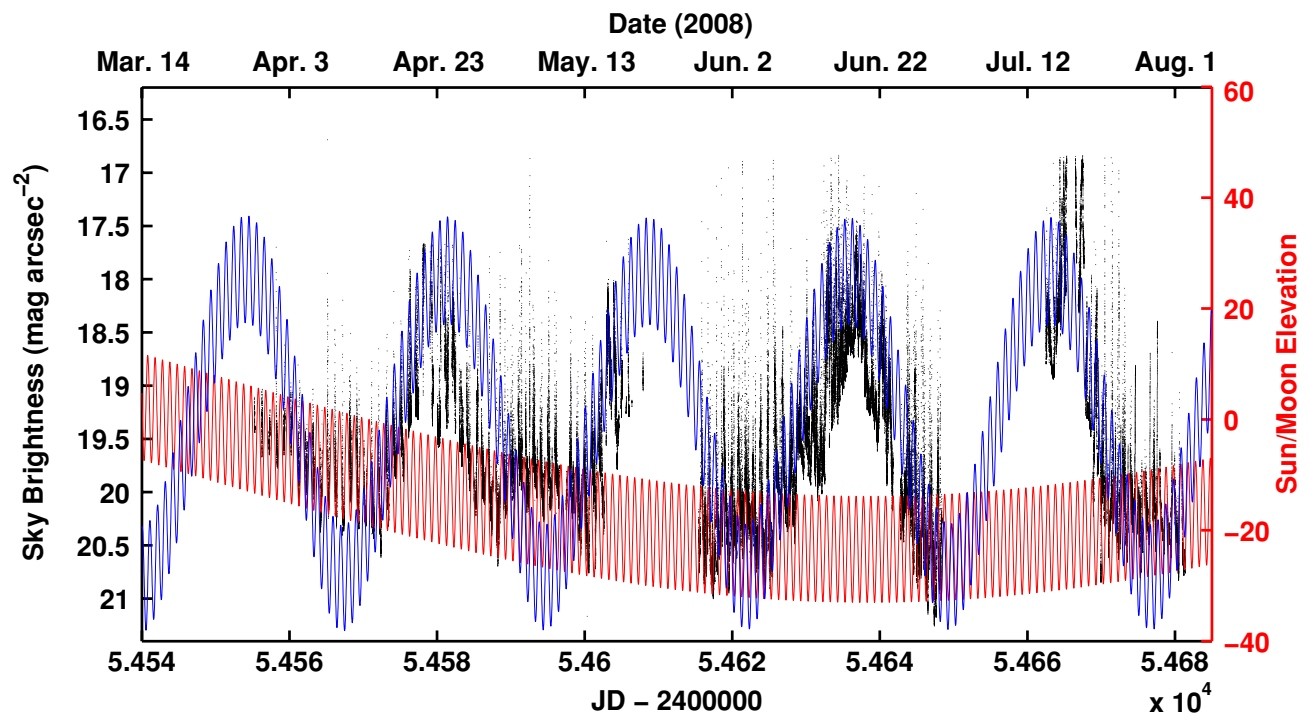

Figure 1. Sky brightness in $i$ band (black dots). The red and blue curves are the elevations of the Sun and the Moon, respectively.
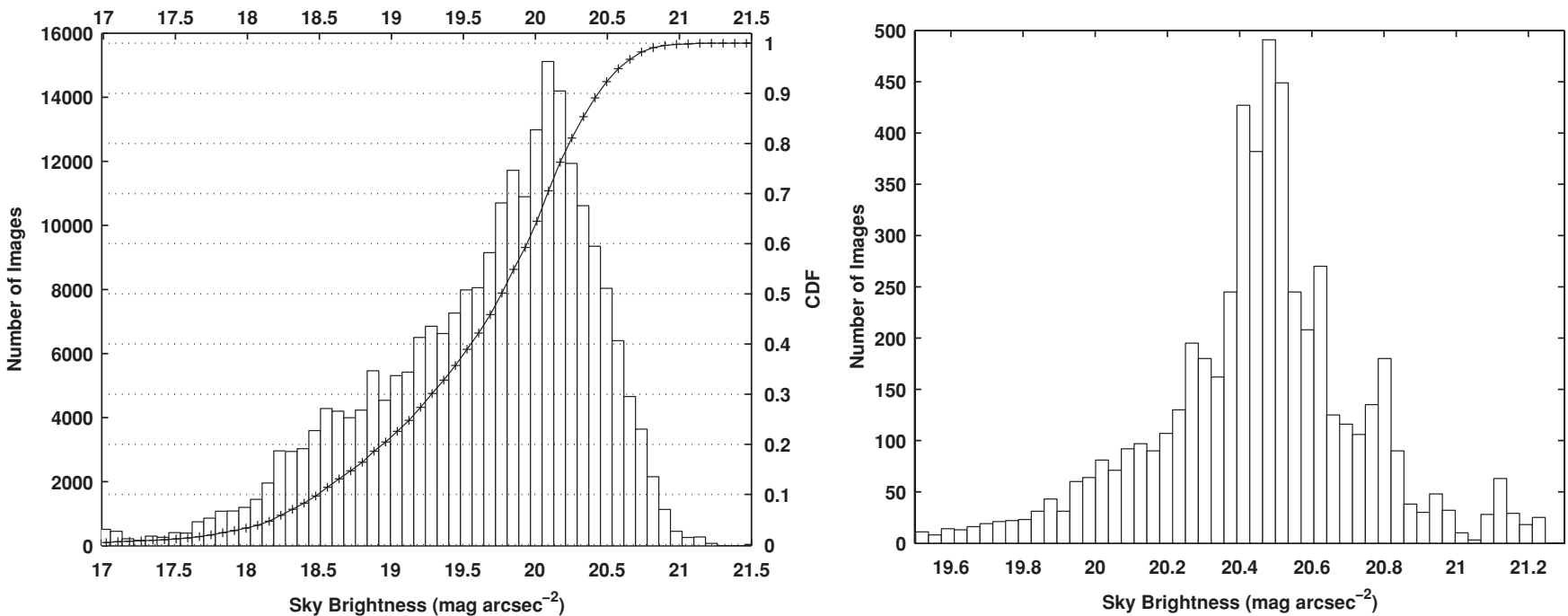

Figure 2. Left panel shows the histogram and the cumulative distribution function (CDF) of the $i$-band sky brightness distribution at Dome A during 2008. Right panel is the same information for the subset of images taken on moonless clear nights in 2008 June.

a calibration constant of $\overline{i_{\mathrm{CSTAR}}-i_{\mathrm{USNO}}}=4.16 \pm 0.12$. SDSS $i$-band magnitudes are very close to $\mathrm{AB}$ magnitudes (Bohlin et al. 2001), so we can transform our $i$-band magnitudes into a top-of-the-atmosphere flux. The magnitudes in the AB system (Oke \& Gunn 1983) are defined as follows:

$$
m=-2.5 \log F_{v}-48.6,
$$

where $m$ is the $\mathrm{AB}$ magnitude and $F_{\nu}$ is the flux of the specified wavelength band in the units of erg s $\mathrm{sm}^{-1} \mathrm{~Hz}^{-1}$. Then we can convert from CSTAR raw ADU counts (normalized to a $20 \mathrm{~s}$ exposure) to an $i$-band sky flux per square arcsec using

$$
F=\mathrm{ADU} \times 10^{-27.798} / 15^{2}=0.708 \times 10^{-30} \mathrm{ADU},
$$

where we have assumed that the typical atmospheric extinction in clear photometric nights is about $0.05 \mathrm{mag} /$ airmass at $i$, taken from the observational results of the Carlsberg Meridian Telescope (CMT) at La Palma, and $15^{2}$ is the pixel area in square arcseconds.
The surface fluxes in the rest of this paper are expressed in units of $10^{-30} \mathrm{erg} \mathrm{s}^{-1} \mathrm{~cm}^{-2} \mathrm{~Hz}^{-1} \operatorname{arcsec}^{-2}$.

Figure 1 shows the sky brightness variations throughout the whole observing period of 2008. A diurnal cycle is evident even in midwinter. At such far-southerly latitudes as Dome A, the Moon is always fairly full whenever it is above the horizon from April to August, creating a strong correlation between lunar elevation (including the correction for parallax) and sky brightness.

Figure 2 shows histograms of the sky brightness distribution at Dome A. The most probable sky brightness, across all lunar phases (left panel of the Figure), is found to be $20.1 \mathrm{mag} \mathrm{arcsec}{ }^{-2}$ and the median is $19.8 \mathrm{mag} \operatorname{arcsec}^{-2}$. About $80 \%$ of the images have sky backgrounds darker than $19 \mathrm{mag} \operatorname{arcsec}^{-2}$.

The right panel of Figure 2 shows the sky brightness distribution of images taken only on moonless and clear nights during 2008 June. In these images, the Sun and the Moon elevation is below $-18^{\circ}$ and there are no clouds. We find that the median sky brightness is $20.5 \mathrm{mag} \operatorname{arcsec}^{-2}$. When it is dark but cloudy, the sky brightness increases to about $20.2 \mathrm{mag} \operatorname{arcsec}^{-2}$. 


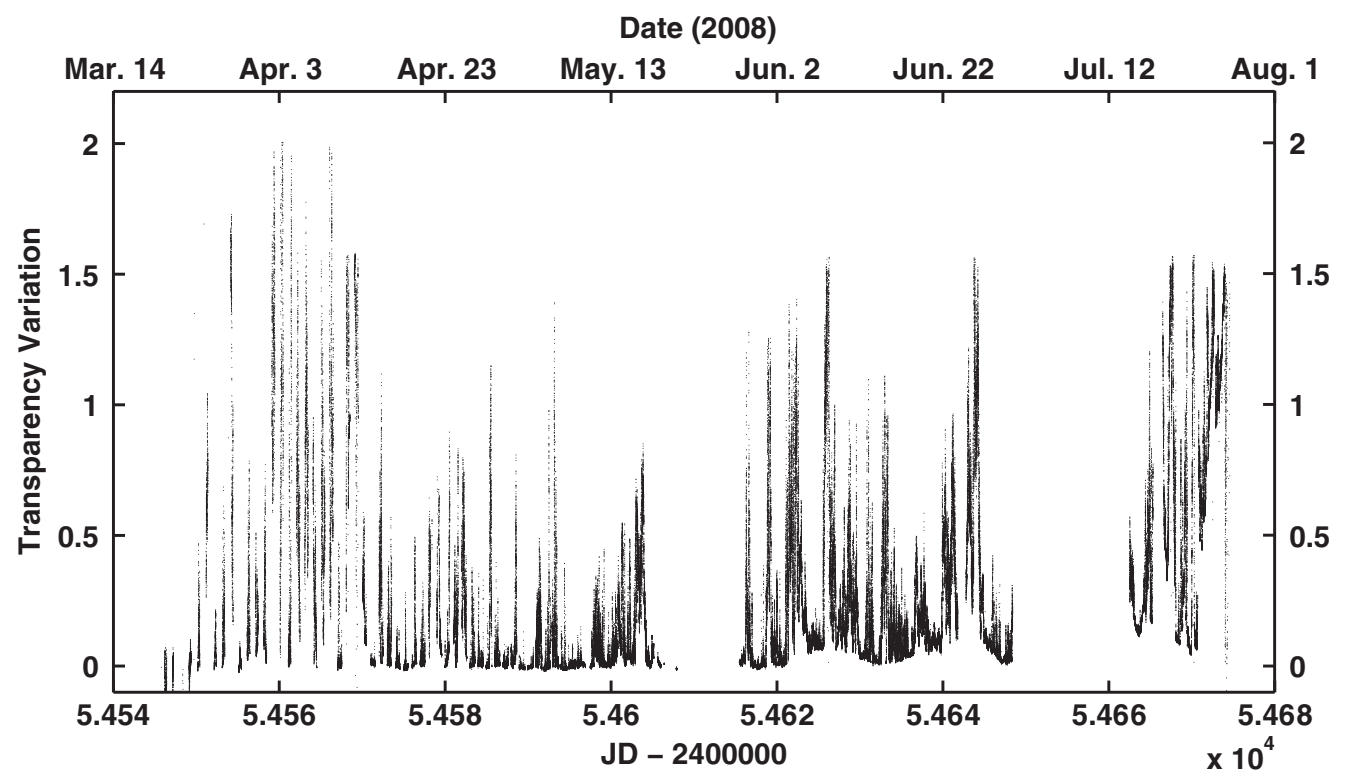

Figure 3. $i$-Band sky transparency variation (in magnitudes) vs. Julian Date (JD) for all the CSTAR images during 2008.

When the Moon is full and it is clear, the brightness is around $19.1 \mathrm{mag} \operatorname{arcsec}^{-2}$. When the Moon is full and there are clouds, the average sky background is $17.9 \mathrm{mag} \operatorname{arcsec}^{-2}$.

Moles et al. (2010) have summarized the night sky brightness in $U B V R I$ measured at various observatories during dark time. We used the transformation equations (Jordi et al. 2006) between SDSS magnitudes and $U B V R I$ to compare our $i$-band measurements with the sky backgrounds of La Palma, Cerro Tololo, and Paranal with Dome A. These transformations, which are appropriate for stars, are slightly different from those that should be used for the sky background because of the presence of strong emission lines. We calculate the following median $i$-band sky brightnesses: 20.10 mag $\operatorname{arcsec}^{-2}$ at La Palma (at sunspot minimum; Benn \& Ellison 1998b), 19.93 at Paranal (at sunspot maximum; Patat 2003), 20.07 at Cerro Tololo (at sunspot minimum; Walker 1987, 1988) and 19.57 at Calar Alto (at sunspot maximum; Sánchez et al. 2007). The CSTAR measurements reported here, with a clear dark sky brightness of $20.5 \mathrm{mag} \operatorname{arcsec}^{-2}$, support the tentative conclusion that under moonless clear conditions, Dome A has a darker sky background than the above astronomical sites, even allowing for calibration and transformation uncertainties of up to several tenths of a magnitude.

\subsection{Correction for Galactic Background}

To compare our observations of the sky background at the south celestial pole with observations taken well away from the Galactic Plane we need to estimate the contribution from diffuse Galactic light. The Pioneer 10 and 11 spacecraft made observations of the total Galactic plus extragalactic sky background in blue (395-485 nm) and red (590-690 nm), from beyond the asteroid belt. The results for selected fields, including the south celestial pole, are given in Toller et al. (1987). The Pioneer values have a resolution of about $2^{\circ}$ on the sky and include the light from stars fainter than about $V \sim 8$. We extrapolate the Pioneer red data into the $i$ band by using the ratio of line-of-sight integrated starlight (Mattila 1980) and the ratio of line-of-sight extinction at the different wavelengths. This leads to an estimate of $(26 \pm 8) \times 10^{-10} \mathrm{erg} \mathrm{cm}^{-2} \mathrm{~s}^{-1} \mathrm{sr}^{-1} \mathrm{~nm}^{-1}$, which is a 23.75 mag contribution to our median of $20.5 \mathrm{mag}$ (i.e., $\sim 5 \%$ of the total sky background). In other words, we would expect the median sky brightness in regions well away from the Galactic plane to be a further $\sim 60$ mmag fainter.

\subsection{Transparency Variations}

Starlight is attenuated as it passes through Earth's atmosphere due to molecular absorption and scattering from molecules and aerosols. Ideally, there would be stable, dry air above the site and, in the absence of clouds, the observed flux of each non-variable star would show little change. Normally, it would be possible to derive the absolute extinction for the site by observing the same star at two very different airmasses or by observing stars of known brightness that happen to be at two different airmasses. However, in the case of CSTAR, with only a 4.5 field, neither technique is practical. We thus confine our discussion to variations in transparency, with the absolute value of the extinction yet to be determined, although presumably it is low at Dome A due to the altitude of the site and the low aerosol content of the atmosphere.

To assess the variations in transparency, we compared the observed brightness of an ensemble of bright, unsaturated, stars to the brightness of these stars on a reference image, chosen as the one containing the greatest number of detected point sources. All images were scaled to the same exposure time, $20 \mathrm{~s}$.

Figure 3 presents the time variation of atmospheric transparency during the periods of darkness throughout 2008, while Figure 4 shows an expanded version covering 10 day periods in each month from April to July. From April 15 to June 2, the transparency was relatively stable with many days showing high transparency. In June and July, the sky reaches its darkest due to the low elevation of the Sun; the transparency became a little worse in July. The median values of the excess extinction, above that of our nominally clear reference image, for the months from April to July are $0.056,0.016,0.127$, and 0.49 , respectively.

Figure 5 shows the distribution of the transparency. Most of the images have only a small increase in transparency relative to the reference image: $90 \%$ of the images have less than 0.7 mag of transparency, $80 \%$ have less than $0.4 \mathrm{mag}$, and more than half have less than $0.1 \mathrm{mag}$. Ivezić et al. (2007) show that in conditions of up to 1 mag of reduced transparency, 


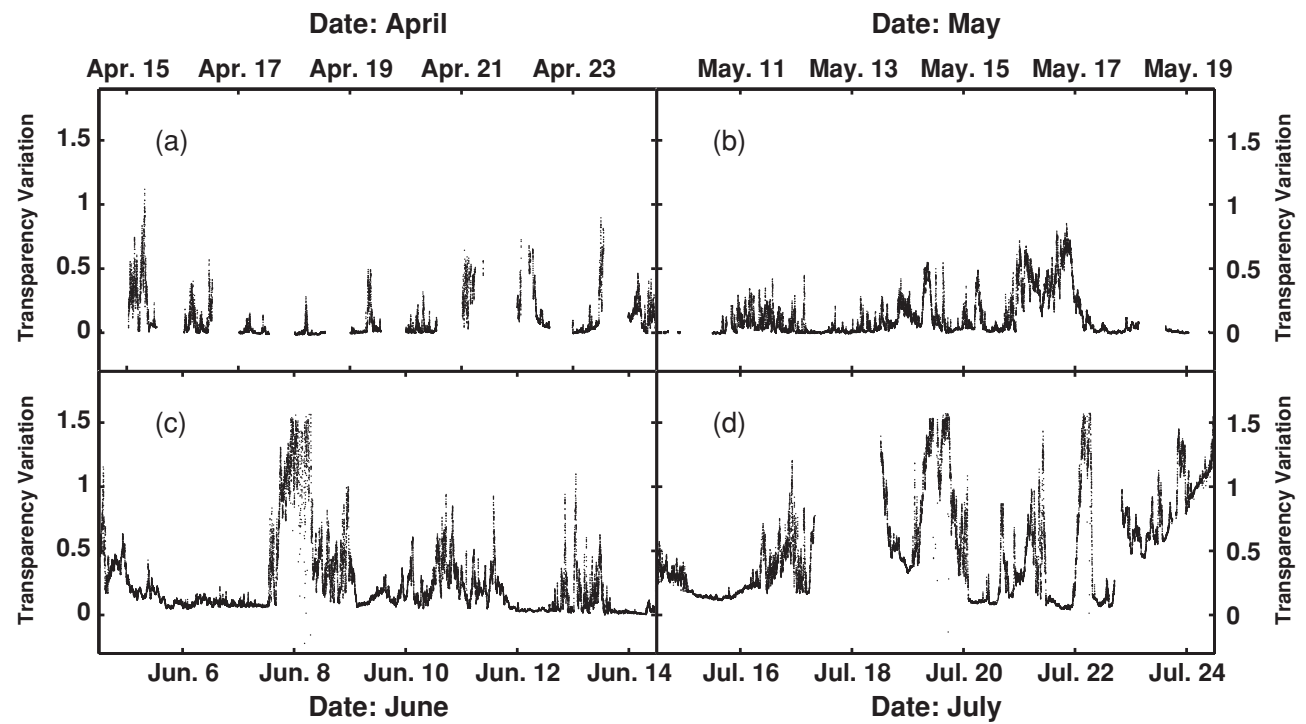

Figure 4. (a) $i$-Band sky transparency variations (in magnitudes) from April 15 to April 25. The gaps are periods when sunlight made the sky too bright to observe. (b) May 10 to May 20, (c) June 5 to June 15 , and (d) July 15 to July 25.

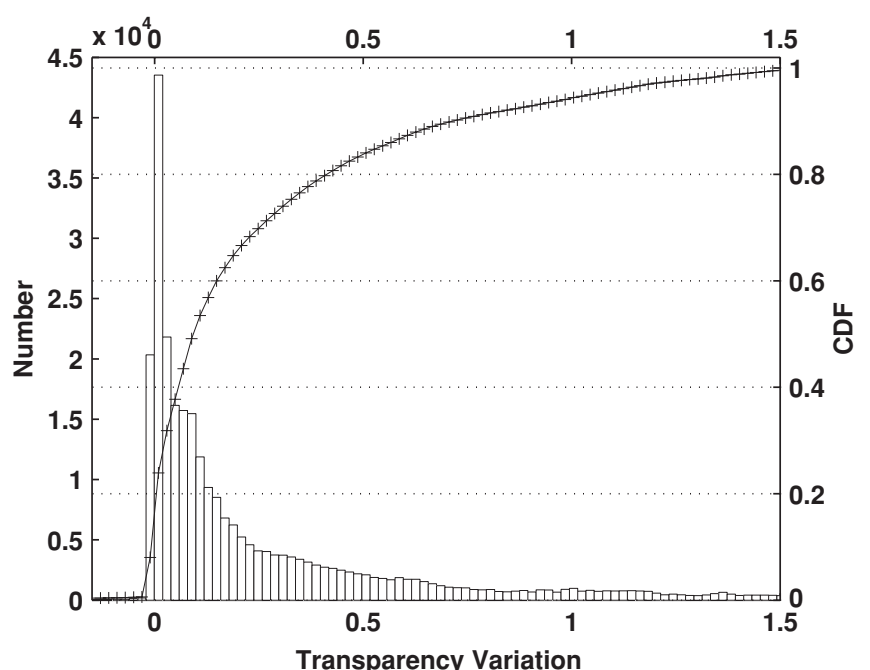

Figure 5. Histogram of the number of images with the given transparency variation (in magnitudes) relative to the reference image. The curved line marked "+" is the CDF.

photometry is limited by photon noise, not by variations in the cloud extinction itself. Therefore, conditions were suitable for accurate photometry for $>90 \%$ of the time during 2008 at Dome A.

\subsection{Percentage Cloud Cover}

Here, we use our observed relative transparency variations to infer the distribution of the optical thicknesses of cloud cover at Dome A during 2008. We note that any reduction in transparency could be due to combinations of clouds, the atmosphere, possible ice formation on the entrance pupil of the telescopes, and the exhaust plume produced by the diesel generators (about $45 \mathrm{~m}$ away from CSTAR). Our results therefore represent upper limits to the cloud cover. We expect window frosting to be negligible, due to the design of the front window of the telescope which is heated throughout the time of observation. Table 1 lists the percentages of images in various extinction ranges - chosen to correspond to the flux percentage of stars relative to the flux in the reference image as shown in the first column. About $51 \%$ of the images were obtained in excellent conditions with excess extinction from any cause of less than $0.11 \mathrm{mag}$.

For a rough comparison with another observatory, Table 2 shows the approximate fraction of clouds of various thicknesses at Mauna Kea. The data for the table come from the Gemini Observatory ${ }^{14}$ and are based on nightly logs from the United Kingdom Infra-red Telescope over a 10 year period. The relative transmission levels Gemini provides are in $V$-band magnitudes (these levels are subjectively assumed by them for the purpose of integration time calculation). We use the calculated atmospheric extinction (King 1985) to convert these $V$-band extinction in clear weather to the $i$ band by simply applying the differential extinction between these two passbands ( $V-i=0.07$ from the observational results of the CMT for La Palma $)^{15}$. Here, $V-i=0.07$ is just like a systematic zero point to transform the cloud extinction ranges of different cloud categories between these two bands, although it is unclear about how the cloud extinction varies in different passbands and from site to site. Table 2 shows that the cloud cover at Dome A falls into the "cloudy" or worse category for a mere $2 \%$ of the time, compared with $30 \%$ at Mauna Kea. There are patchy clouds at Dome A for about $31 \%$ of the total usable time, most of which are cirrus clouds passing by as verified by wide-field cameras on the top of PLATO when the Moon is up. Overall there is a greater fraction of photometric conditions at Dome A compared to Mauna Kea. However, these conclusions are tentative due to the limited sky field of CSTAR, different photometric systems, and the fact that the Dome A data are from only 5 months during 2008.

\subsection{Absolute Flux from the Sun}

The Sun and the Moon have of course a strong effect on the sky background, especially when they are above the horizon. Liu et al. (2003) modeled the relationship between the sky brightness and the phase and elevation of the Moon. The way in which the Sun and the Moon affect the sky background should be formally

\footnotetext{
14 http://www.gemini.edu/sciops/telescopes-and-sites/ observing-condition-constraints

15 http://www.ast.cam.ac.uk/ dwe/SRF/camc_extinction.html
} 


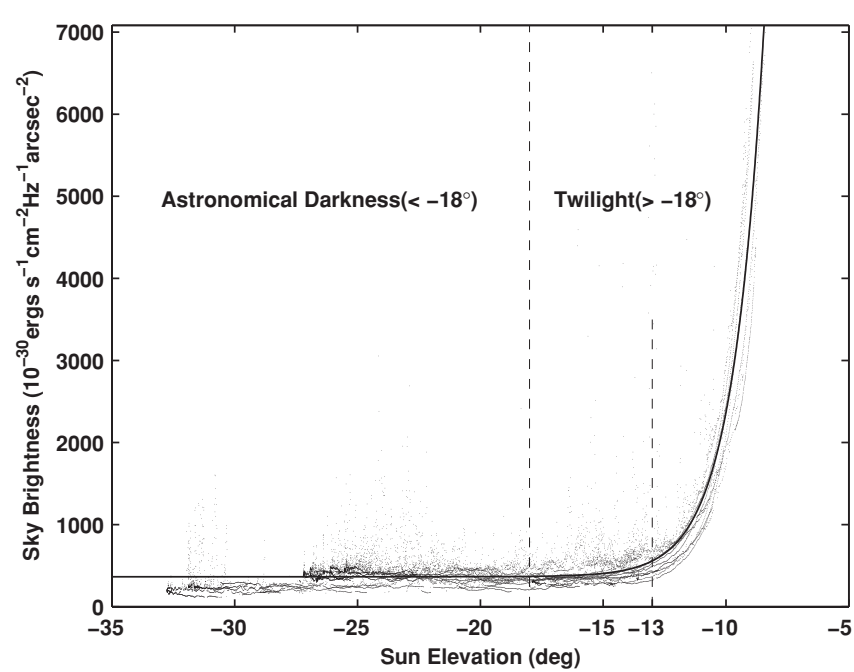

Figure 6. Relation between the sky brightness and the elevation of the Sun. The data points are those images that were taken in good conditions with negligible contribution from the Moon. The curved line is the fit from Equation (2). The dashed line at $-18^{\circ}$ gives the dividing elevation where the astronomical darkness is defined to begin. The dashed line at $-13^{\circ}$ indicates a more suitable cutoff for $i$-band dark time at Dome A.

Table 1

Cloud Cover Conditions at Dome A

\begin{tabular}{cccc}
\hline \hline Flux & Excess Extinction $(\mathrm{mag})$ & Fraction & Cloud Cover \\
\hline$<50 \%$ & $>0.75$ & $9 \%$ & Thick \\
$50 \%-75 \%$ & $0.31-0.75$ & $17 \%$ & Intermediate \\
$75 \%-90 \%$ & $0.11-0.31$ & $23 \%$ & Thin \\
$>90 \%$ & $<0.11$ & $51 \%$ & Little or none \\
\hline
\end{tabular}

Notes. The first column is the observed flux of our standard stars relative to the reference image. The second column gives the $i$-band magnitude change (excess extinction) corresponding to the "flux." The last column gives the description of the cloud cover.

Table 2

The Comparison of Cloud Cover Between Mauna Kea and Dome A

\begin{tabular}{lccc}
\hline \hline & Mauna Kea (Gemini) & & Dome A \\
\hline Cloud Cover & Extinction $(V)$ & Fraction & Fraction \\
\hline Any other usable & $>3$ & $10 \%$ & 0 \\
Cloudy & $2-3$ & $20 \%$ & $2 \%$ \\
Patchy cloud & $0.3-2$ & $20 \%$ & $31 \%$ \\
Photometric & $<0.3$ & $50 \%$ & $67 \%$ \\
\hline
\end{tabular}

Notes. The definition of cloud cover is adopted from the Gemini Observatory. For comparison, we use $V-i=0.07$ in extinction for the different transparencies of these two bands as presented in the text. Note that the term "photometric" as used here is just one kind of cloud cover category and it is different from the normal term "photometric night."

analogous except for the additional complication that the Moon exhibits phases.

Referring to the model of Liu et al. (2003) in $V$ band, we fit the sky background flux as a function of the solar elevation with the same formula for our $i$-band data,

$$
F_{\text {Sun }}=a 10^{b \theta}+c,
$$

where $F_{\text {Sun }}$ is the sky flux (assuming only a solar contribution and a background), $\theta$ is the elevation of the Sun, and $a, b$ and $c$ are constants. We fitted for the constants with a nonlinear leastsquares method, using the images with good transparency and no contribution from the Moon. The result is

$$
F_{\text {Sun }}=4.906 \times 10^{6} \times 10^{0.339 \theta}+370
$$

and is plotted against the observations in Figure 6. We find that the sky is about as dark as it ever gets in the $i$ band once the Sun is below about $13^{\circ}$ from the horizon.

\subsection{Astronomical Twilight and Hours of Dark Time}

Astronomical twilight is defined as the time when the Sun is between $12^{\circ}$ and $18^{\circ}$ below the horizon. However, this definition is really only appropriate at low altitude observatories and in the $V$ band. At the high elevation of Dome A, and in the $i$ band, the sky is as dark as it ever gets when the solar elevation reaches about $-13^{\circ}$ (see Figure 6). Table 3 summarizes the numbers of hours when the Sun is located below elevations of $-18^{\circ}$, $-13^{\circ}$, and $0^{\circ}$. If we require only that the Sun is below the horizon, Dome A has more "night time" that any of the other sites. This is because Earth's elliptical orbit around the Sun results in the total hours of night time in a year increasing with distance from the north pole. However, for "dark time" to occur, we require the Sun to be at least a certain elevation below the horizon (conventionally, $-18^{\circ}$ ). There is less dark time at Dome A compared to temperate-latitude observatories, although the $-13^{\circ}$ solar elevation limit makes this difference less dramatic than it would be at $-18^{\circ}$. While the total dark time is less, the long periods of continuous darkness are an advantage for many observations. Over $83 \%$ images were obtained during 2008 when the Sun was $13^{\circ}$ below the horizon. The discussion in the rest of this paper is based on these images.

\subsection{Absolute Flux from the Moon}

The model for the sky surface brightness caused by moonlight is more complex. In our analysis, we ignore the variation in the lunar brightness caused by the changes in the Moon-Earth distance. Following Liu et al. (2003), the apparent magnitude of the Moon can be approximated by an empirical formula:

$$
V(R, \Phi)=0.23+5 \log R-2.5 \log P(\Phi),
$$

where $R$ is the Moon-Earth distance, $\Phi$ is the Moon phase angle, and $P(\Phi)$ is the function of the full Moon illuminance. Then, using the same approach as for the Sun, the sky surface flux contribution by the Moon, $F_{\text {moon }}$, can be expressed in the form of Equation (1) multiplied by $P(\Phi)$. Then,

$$
F_{\text {moon }}=A P(\Phi) 10^{B \Theta}+C
$$

Table 3

Hours Per Year at Different Sites When the Sun is Below a Given Elevation

\begin{tabular}{lccccc}
\hline \hline \multicolumn{1}{c}{ Site } & Latitude & Elevation $(\mathrm{m})$ & Hours $\left(<-18^{\circ}\right)$ & Hours $\left(<-13^{\circ}\right)$ & Hours $\left(<0^{\circ}\right)$ \\
\hline Dome A (Antarctica) & $-80^{\circ} 22^{\prime}$ & 4093 & 1680 & 2606 & 4471 \\
Mauna Kea (Hawaii) & $+19^{\circ} 50^{\prime}$ & 4194 & 3395 & 3689 & 4394 \\
Cerro Pachón (Chile) & $-30^{\circ} 14^{\prime}$ & 2722 & 3330 & 3640 & 4404 \\
La Palma (Spain) & $+28^{\circ} 46^{\prime}$ & 2332 & 3313 & 3623 & 4381 \\
\hline
\end{tabular}




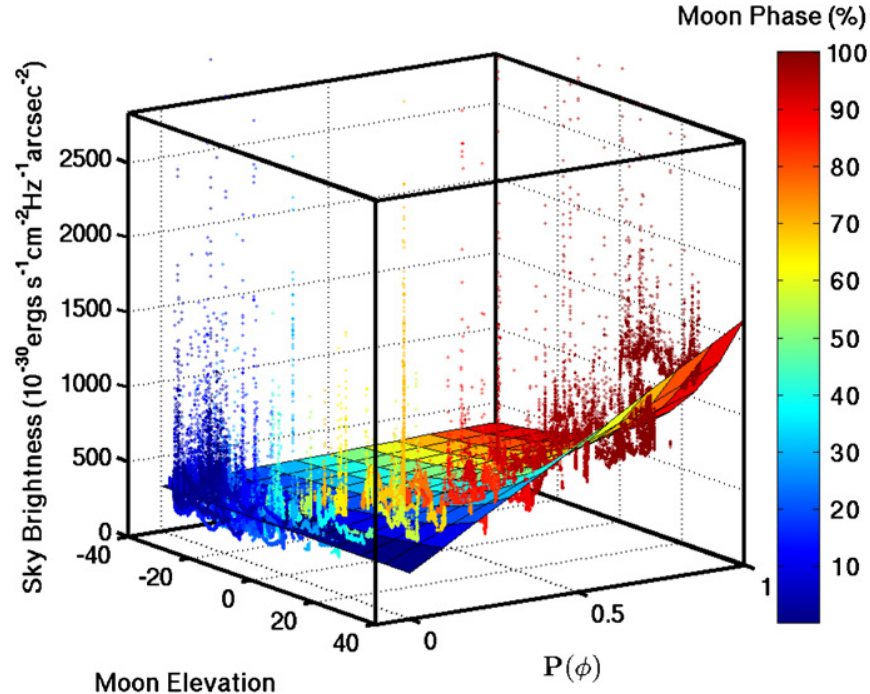

Figure 7. Relationship among the sky brightness (with the solar contribution subtracted and under conditions of good relative transparency), the lunar phase, and the elevation of the Moon. The curved and colored surface is fitted by the model in the form of Equation (3). The color bar is related to the Moon phase in percentage, which is the illuminated fraction of the full Moon disk. The outliers may be aurorae.

where $\Theta$ is the elevation of the Moon and $A, B$, and $C$ are constants. $C$ is the intrinsic background flux to be fitted. We applied these equations to the $i$-band data. Although the model is somewhat crude, it works well in the estimation of sky brightness illuminated by the Moon. For a more refined but slightly more complicated sky brightness model, we can refer to the study of Krisciunas \& Schaefer (1991).

Figure 7 displays the Moon contribution to sky surface brightness as the function of the Moon phase and elevation. The sky brightness in the figure is the Sun-corrected sky surface flux without any contribution from clouds. The colored curved surface is the fitted model described by

$$
F_{\text {moon }}=300.576 P(\Phi) 10^{0.0162 \Theta}+306.813 \text {. }
$$

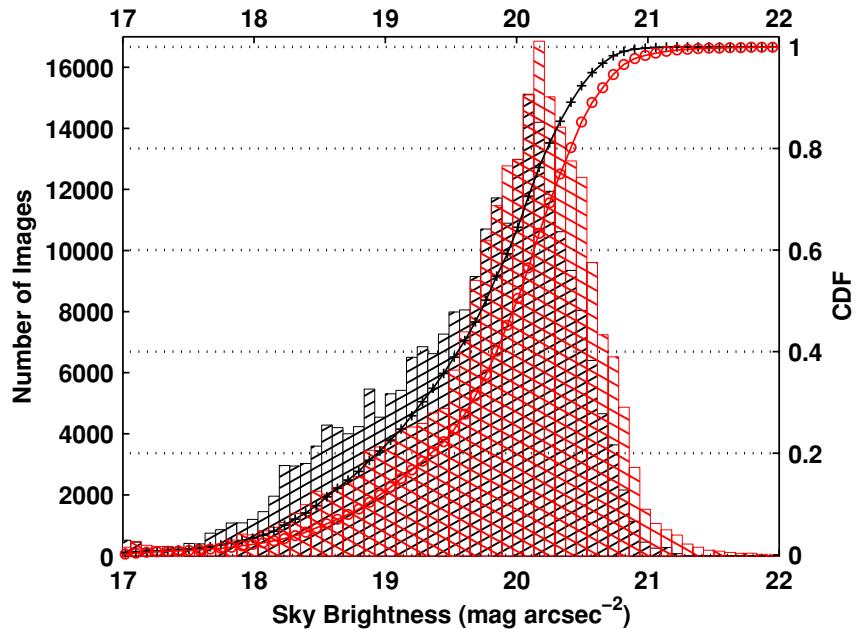

Figure 9. Histograms of the sky brightness distributions of both the original data and the modified one (subtracting the Sun and Moon contributions). The area patched with black slashes and the curved line with markers " + " represent the raw sky brightness and its CDF. The area with red backslashes and the curved line with circles are the modified sky background and its CDF.

We can see that the sky was brightest when the Moon was almost full. The outliers should be the points when aurorae were present.

We compare the modified sky background (removing both the solar and lunar contributions) with the raw one (before removing the solar contribution) in Figure 8. The modified sky background becomes flat wherever the Moon is up. Figure 9 compares the histograms of the raw sky brightness in units of mag $\operatorname{arcsec}^{-2}$ (the left panel shown in Figure 2) with the modified sky background. The histogram with black slashes represents the original data and the one patched with red backslashes is for the modified sky brightness. The median sky brightness becomes $20.04 \mathrm{mag} \operatorname{arcsec}^{-2}$ after being corrected. About $80 \%$ of the images are fainter than 19.35 mag $\operatorname{arcsec}^{-2}$.

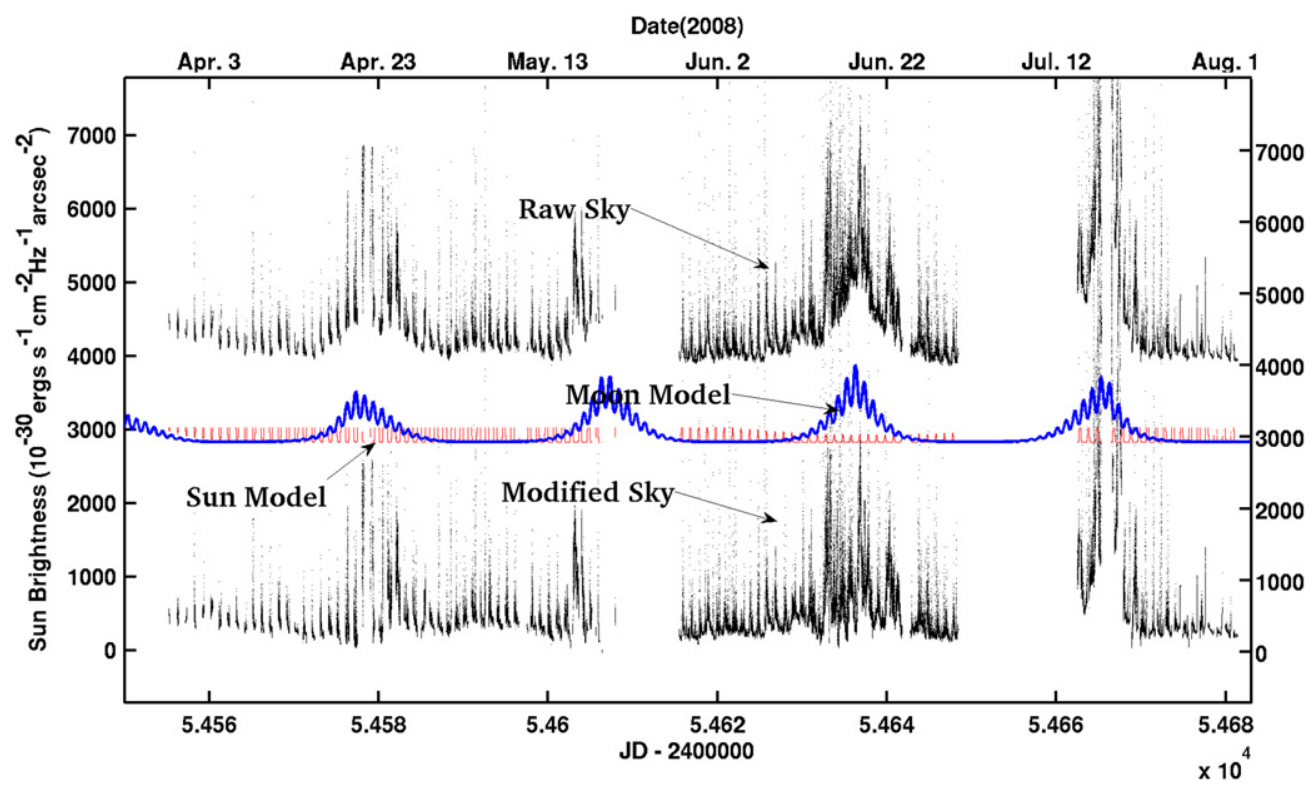

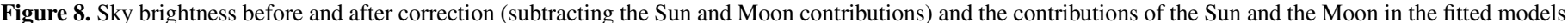

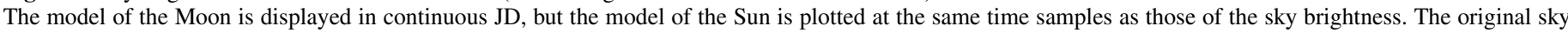
brightness and the model fluxes of the Sun and the Moon are shifted vertically from their proper values. 


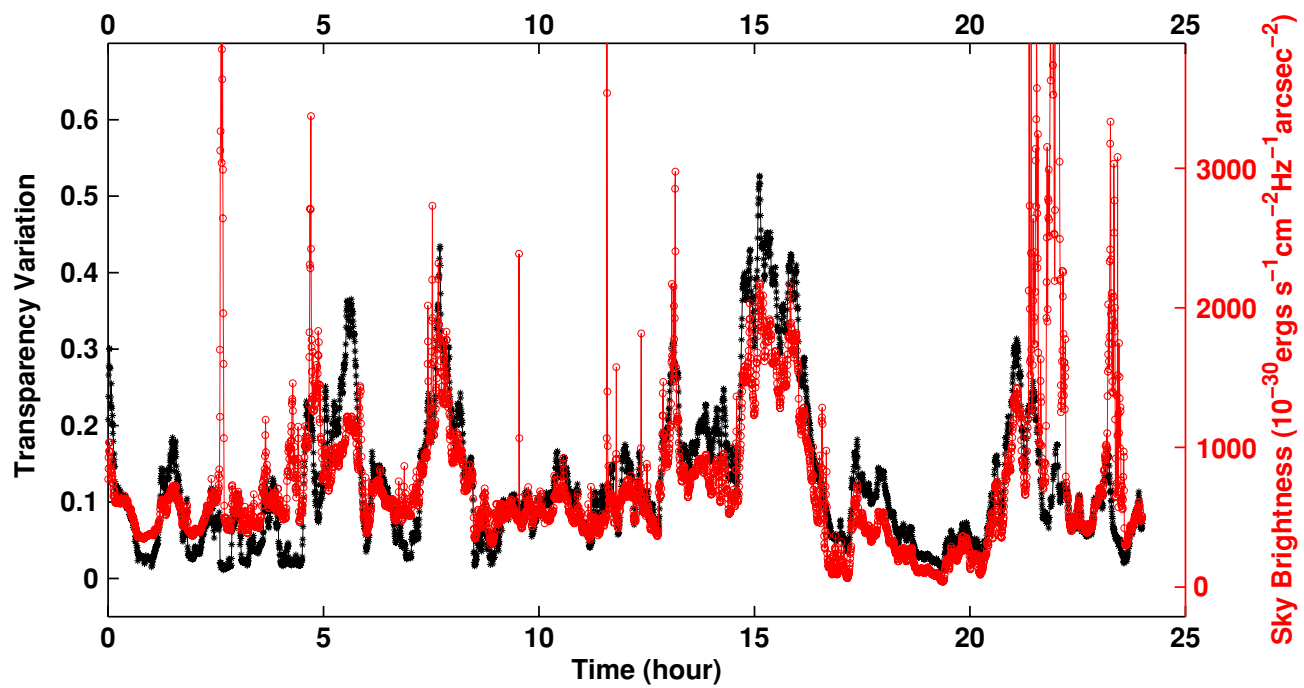

Figure 10. Variations of the sky brightness and transparency variation (excess extinction in magnitudes) for 1 day in 2008. The black asterisks are the excess extinction and the red circles are the sky background. The $x$-axis is the time in hours elapsing from 2008 June 16 00:00 UTC.

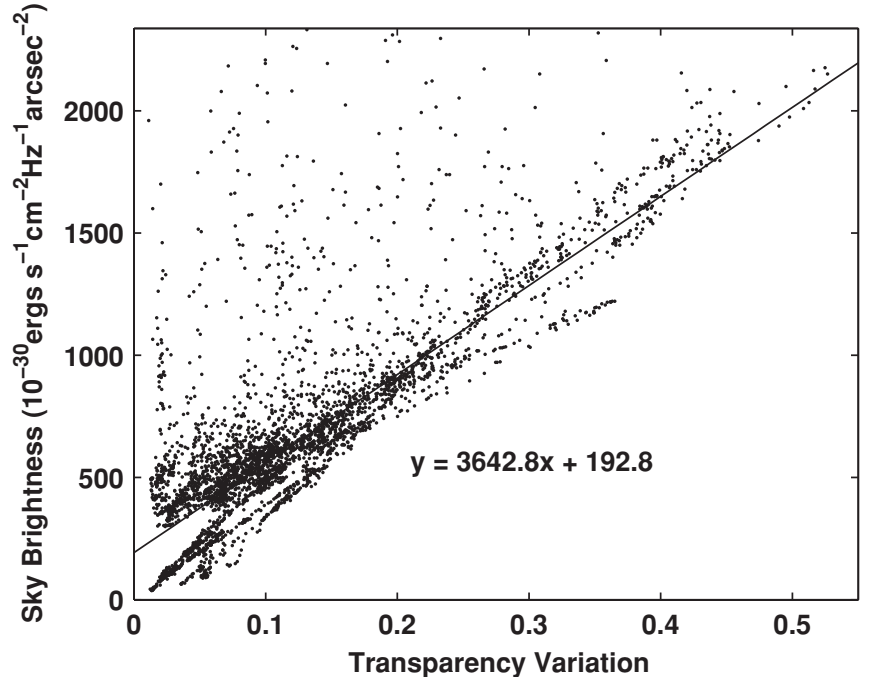

Figure 11. Correlation of the corrected sky brightness and transparency variation (in magnitudes). The data points are observations taken on 2008 June 16, when the average elevations of the Sun and the Moon are -23.17 and $25^{\circ} .12$, respectively, and the lunar phase was $\sim 95 \%(\Phi=25.8, P(\Phi)=0.54)$. The straight line is the fitted relation with the equation shown.

\subsection{Correlation Between Sky Brightness and Transparency Variation}

The sky will be brighter when there is scattering of the Sun and the Moon light by clouds. In order to study this effect, we choose a day, June 16, when the Moon was nearly full, the Sun was far below the horizon, and the transparency was varying. Figure 10 shows the variations of the sky brightness and relative transparency in this day. We can see that they are strongly correlative and the plot of the sky brightness against transparency in Figure 11 confirms that the relationship is linear (again, outliers are the data polluted by aurorae). The line of best fit and the derived linear equation are also shown in the figure.

The sky brightness is proportional to both the extinction (here, relative transparency) and the total illuminance of the Sun and the Moon. The larger the extinction, the brighter the sky background for a given total illuminance. The greater the total illuminance, the brighter the sky background for a given extinction.

We construct a model describing the sky brightness as a function of the total illuminance and transparency variation. When the relative transparency is negligible, the sky background should be the intrinsic sky background (a constant value), resulting in the model not containing an individual term of the Sun and the Moon. At new Moon or when the Moon is low enough below the horizon and the elevation of the Sun is low, the sky brightness should be the sum of the intrinsic background and cloud contribution. This requires a single relative transparency term. Then

$$
F_{\text {sky }}=a\left(F_{\text {sun }}+F_{\text {moon }}\right) E+b E+c,
$$

where $F_{\text {sky }}$ is the corrected flux of the sky light, $F_{\text {sun }}$ and $F_{\text {moon }}$ are the model fluxes of the Sun and the Moon given by Equations (2) and (4) (without constant terms), $E$ is the relative transparency and, $a, b$ and $c$ are constants. $c$ denotes the fitted intrinsic background and $b$ should be less than zero, indicating that the sky is dimmed by the clouds when it is a moonless night and the Sun is very low. The result, which gives an excellent fit to the data, is

$$
F_{\text {sky }}=5.4751\left(F_{\text {sun }}+F_{\text {moon }}\right) E-93.98 E+291.96 \text {. }
$$

\subsection{Percentage of Images Affected by Aurorae}

For our SDSS $i$ band (effective wavelength: $780 \mathrm{~nm}$; bandwidth: $160 \mathrm{~nm}$; Zhou et al. 2010a), the emissions of aurorae within this band mainly contain the lines from molecular nitrogen and molecular oxygen in the lower atmosphere and those from atomic oxygen in the higher atmosphere. The typical green-yellow auroral emission lines are dominated by the atomic oxygen, lying outside the $i$ band (Dempsey et al. 2005). To estimate the percentage of images affected by aurorae, we use the model of sky brightness as described in Equation (5). Figure 12 shows how well the model fits the data and identifies the outlying points (implying the presence of aurorae) as circles. We define an outlier-and hence the image affected by an aurora - as a point that is more than $3 \sigma$ brighter than the model would predict (although it is also possible that a small fraction of those images are affected by airglow). 


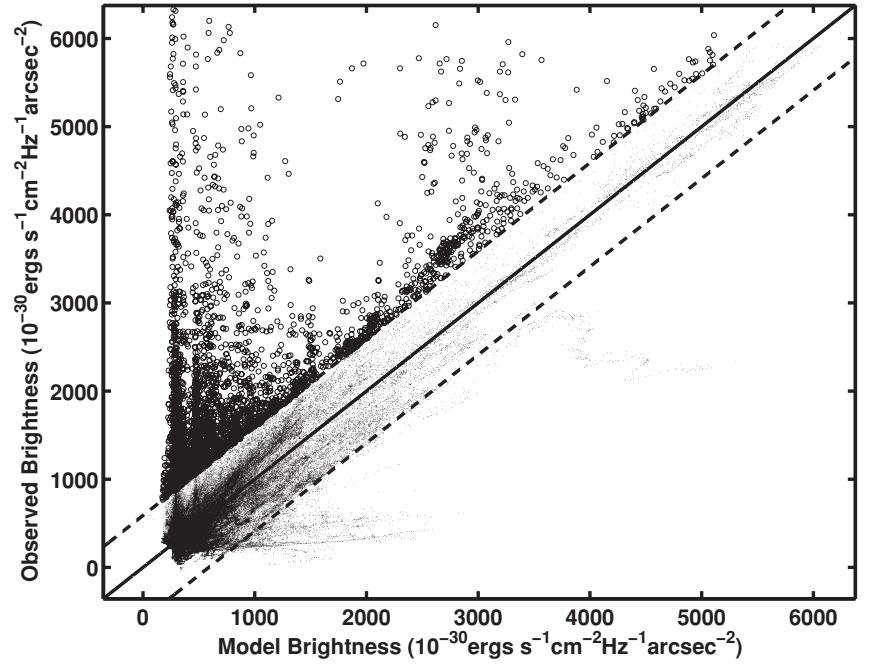

Figure 12. Observed sky brightness corrected for the Sun and Moon contributions vs. the values predicted by the fitted model from Equation (5). The diagonal denotes where the observations and model agree exactly. The two dashed lines are $3 \sigma$ above and below this level. The circles show the observed data points, presumably affected by aurorae, with brightness brighter than $3 \sigma$ above the model. The transparency variations are in magnitudes.

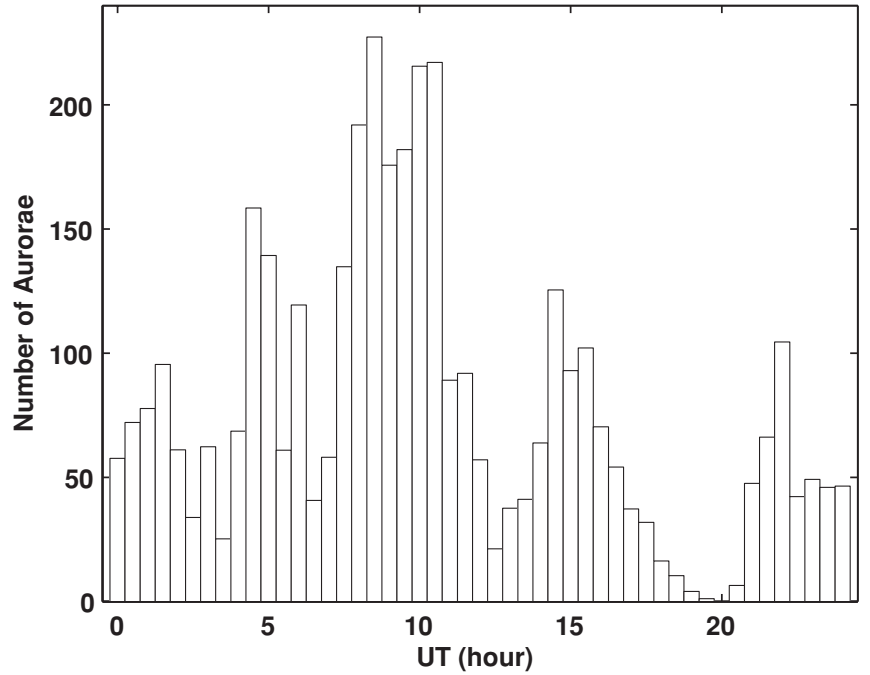

Figure 13. Time distribution of the images taken during 2008 that were affected by aurorae. The counts are corrected for the image-sampling time distribution.

There are about 3800 images polluted by aurorae or less than $2 \%$ of the total. Most of the detected aurorae should be comparatively strong ones. The aurorae with weaker intensities are immersed in the fluctuation of the real observed data and errors of the fitted model. Therefore, the fraction of aurorae is a lower limit. However, we believe that any aurorae possibly present in the remaining $98 \%$ of our images are sufficiently faint as to not affect photometric accuracy. We do not find an obvious dependence of the aurorae on the transparency variation or cloud coverage, which implies that the above aurorae are reasonably detected, because the transparency variation distribution of those polluted images are generally uniform. Figure 13 shows the time distribution of the images affected by aurorae. A considerable number of them are concentrated before or after 7:00 in UT when the Sun is highest. We can also find that there are few or no images affected by aurorae at about 20:00 nearly when the Sun elevation is lowest at 19:00.
An optical spectrometer called Nigel (Kenyon et al. 2006) was installed in PLATO at Dome A in 2009 January and this will allow unambiguous identification of aurorae.

\section{CONCLUSION}

In 2008, CSTAR was deployed at Dome A in Antarctica to measure the night sky background, the weather conditions, and to study variable stars. About 310,000 images were acquired during the observing period of over four months. We have used these data to quantify the $i$-band sky brightness, variations in sky transparency, the background flux from the Sun and the Moon, and to derive some statistics on aurorae.

Due to the high ecliptic latitude of the observed south celestial pole area, the sky background is little affected by the zodiacal light. The $i$-band sky brightness has a median value of 20.5 mag $\operatorname{arcsec}^{-2}$ when not affected by the Sun and the Moon. When corrected for the diffuse Galactic background, the sky brightness drops a further $0.06 \mathrm{mag}$.

Comparing each image to our "best," or reference, image observed on a clear and moonless night, variations in transparency can be calculated. We find that the weather was stable and there was little cloud coverage during most of the observing time. Conditions became somewhat worse and the transparency changed considerably in June and July when the Sun was at its lowest below the horizon in the year. Among the $83 \%$ images in which the elevation of the Sun was lower than $-13^{\circ}$, about $67 \%$ of them were taken under conditions of little or no cloud coverage, using the same criterion for the cloud cover as used by the Gemini Observatory at Mauna Kea.

On the whole, Dome A has excellent optical sky backgrounds and had low cloud fractions during 2008. About 2\% of our images were affected by relatively strong aurorae.

This study has been supported by the Chinese National Natural Science Foundation through grants 10873016, 10803007, 10473012, 10573020, 10633020, 10673012, and 10603006, and by the National Basic Research Program of China (973 Program), No. 2007CB815403. This research is also supported by the Chinese PANDA International Polar Year project and the Polar Research Institute of China (PRIC). The support of the Australian Research Council and the Australian Antarctic Division for the PLATO observatory is gratefully acknowledged. The authors thank all members of the 2008 and 2009 PRIC Dome A expeditions for their heroic effort in reaching the site and for providing invaluable assistance to the expedition astronomers in setting up and servicing the PLATO observatory and its associated instrument suite. Iridium communications were provided by the US National Science Foundation and the United States Antarctic Program. Additional financial contributions have been made by the institutions involved in this collaboration.

We thank K. Mattila for helpful discussions on the diffuse Galactic light at the south celestial pole.

\section{REFERENCES}

Aristidi, E., et al. 2005a, A\&A, 444, 651

Aristidi, E., et al. 2005b, A\&A, 430, 739

Benn, C. R., \& Ellison, S. L. 1998a, New Astron. Rev., 42, 503

Benn, C. R., \& Ellison, S. L. 1998b, La Palma Tech. Note, 115

Bohlin, R. C., Dickinson, M. E., \& Calzetti, D. 2001, AJ, 122, 2118

Bonner, C. S., et al. 2010, PASP, submitted

Burton, M. G., et al. 2005, PASA, 22, 199

Chamberlin, R. A. 2001, J. Geophys. Res., 106, 20101 
Dempsey, J. T., Storey, J. W. V., \& Phillips, A. 2005, PASA, 22, 91

Ivezić, Z., et al. 2007, AJ, 134, 973

Jordi, K., Grebel, E. K., \& Ammon, K. 2006, A\&A, 460, 339

Kenyon, S. L., Ashley, M. C. B., Everett, J. R., Lawrence, J. S., \& Storey, J. W. V. 2006, Proc. SPIE, 6267, 62671M

Kenyon, S. L., \& Storey, J. W. V. 2006, PASP, 118, 489

Kilcik, A., Anderson, C. N. K., Rozelot, J. P., Ye, H., Sugihara, G., \& Ozguc, A. 2009, ApJ, 693, 1173

King, D. L. 1985, La Palma Tech. Note, 31

Krisciunas, K., \& Schaefer, B. E. 1991, PASP, 103, 1033

Lawrence, J. S., Ashley, M. C. B., Tokovinin, A., \& Travouillon, T. 2004, Nature, 431,278

Lawrence, J. S., et al. 2009, Rev. Sci. Instrum., 80, 064501

Lawrence, J. S., et al. 2009, PASA, 26, 379

Leinert, C., et al. 1997, VizieR Online Data Catalog, 412, 70001

Liu, Y., Zhou, X., Sun, W.-H., Ma, J., Wu, H., Jiang, Z., Xue, S., \& Chen, J. 2003, PASP, 115,495

Mattila, K. 1980, A\&AS, 39, 53

Moles, M., Sánchez, S. F., Lamadrid, J. L., Cenarro, A. J., Cristóbal-Hornillos, D., Maicas, N., \& Aceituno, J. 2010, PASP, 122, 363

Oke, J. B., \& Gunn, J. E. 1983, ApJ, 266, 713
Patat, F. 2003, A\&A, 400, 1183

Ruhl, J., et al. 2004, Proc. SPIE, 5498, 11

Sánchez, S. F., Aceituno, J., Thiele, U., Pérez-Ramírez, D., \& Alves, J. 2007, PASP, 119, 1186

Saunders, W., et al. 2009, PASP, 121, 976

Storey, J. W. V., Lawrence, J. S., \& Ashley, M. C. B. 2007, RevMexAA, 31, 25

Storey, J. W. V., Ashley, M. C. B., Burton, M. G., Lawrence, J. S., \& Saunders, W. 2008, in EAS Publ. Ser. 33, 2nd ARENA Conference: The Astrophysical Science Cases at Dome, ed. C. H. Zinnecker, N. Epchtein, \& H. Rauer (Les Ulis: EDP Sciences), 207

Takato, N., et al. 2008, in EAS Publ. Ser. 33, 2nd ARENA Conference: The Astrophysical Science Cases at Dome, ed. C. H. Zinnecker, N. Epchtein, \& H. Rauer (Les Ulis: EDP Sciences), 271

Toller, G., Tanabe, H., \& Weinberg, J. L. 1987, A\&A, 188, 24

Walker, A. 1987, N.O.A.O. Newsletter, No. 10, 16

Walker, A. 1988, N.O.A.O. Newsletter, No. 13, 22

Yang, H., et al. 2009, PASP, 121, 174

Yang, H., et al. 2010, PASP, in press

Yuan, X., et al. 2008, Proc. SPIE, 7012, 70124G

Zhou, X., et al. 2010a, Res. Astron. Astrophys., 10, 279

Zhou, X., et al. 2010b, PASP, 122, 347 